\title{
High-Resolution TEM Analysis of Supported Metal Nanoparticles Combining with Image Simulation
}

\author{
B.S. Zhang ${ }^{1}$, W. Zhang ${ }^{2}$, L.D. Shao ${ }^{2}$ and D.S. $\mathrm{Su}^{1,2}$ \\ 1. Shenyang National Laboratory for Materials Science, Institute of Metal Research, Chinese Academy \\ of Sciences, 72 Wenhua Road, Shenyang 110016, China. \\ 2. Department of Inorganic Chemistry, Fritz Haber Institute of the Max Planck Society, Faradayweg 4-6, \\ Berlin 14195, Germany.
}

Metal nanoparticles (NPs) exhibiting fascinating chemical, physical, optical, electronic, and magnetic properties that are often radically different from their bulk counterparts have received wide recognition due to their unique applications in many fields, such as catalysis, energy storage, and so on [1-5]. In general, the metal NPs are distributed on various supports, such as $\mathrm{Al}_{2} \mathrm{O}_{3}, \mathrm{Ga}_{2} \mathrm{O}_{3}$, and nanocarbon, to obtain good dispersions and large exposed surfaces. The final sizes, shapes, exposed surfaces and structures of supported metal NPs always play a key role in the heterogeneous catalysis process. The high-resolution transmission electron microscopy (HRTEM) techniques can be used to revealing the local and surface structure of metal NPs, especially the state-of-the-art spherical aberration corrected TEM and scanning TEM are capable of yielding the structures of ultra-fine NPs and clusters. However, since the HRTEM image is a two-dimensional (2D) representation of an originally three-dimensional (3D) object, it is hard to explore the fine structures of terminated surface, facet edges and corners of 3D metal NPs. Electron tomography is a powerful technique for reconstructing 3D structure of an object from a tilt series of 2D images, but it is usually not suitable for small metal NPs and electron-beamsensitive NPs. Alternately, the image simulation is a good supplementary method for revealing the fine structures of metal NPs [4-6].

The idea of simulating HRTEM images arose because of the realization that the loss of phase information when we form an experimental image means that we cannot go back from the image to the real shape and structure of metal NPs. Therefore, the image simulation work is very important for understanding and interpreting the experimental images of metal NPs. There are several methods to determine the fine structure from HRTEM image, such as reconstruction of electron exit wave and deconvolution methods; each has advantages and disadvantages. The purpose of these methods is making the simulated image and experimental image match well enough. In our current work, we selected $\mathrm{Au}$ NPs supported on active carbon $(\mathrm{Au} / \mathrm{AC})$ and Pd NPs supported on carbon black $(\mathrm{Pd} / \mathrm{CB})$ as model materials. After obtaining HRTEM images of these metal NPs by using spherical aberration corrected TEM, we perform the image simulation for interpreting the experimental images connected with their properties. Figure 1shows the HRTEM image of $\mathrm{Pd} / \mathrm{CB}$ and corresponding simulated image and structural model of Pd NP. Combining with image simulation, we can conclude that this Pd NP is a single twinned Pd NP along [123] direction. The HRTEM image matches well with the simulated [123] projected Pd NP image. Moreover, the 3D shape and exposed facets of Pd NP can be identified by the simulated 3D model. Similarly, Figure 2 shows the experimental and simulated images of a multiplytwinned $\mathrm{Au} \mathrm{NP}$ supported on active carbon, which give the detailed 3D information of a truncated decahedron Au NP.

In summary, these experiments show that the fine 3D structure of metal NPs can be interpreted by HRTEM images combining with image simulation, suggesting that a new pathway of the HRTEM 
analysis of metal NPs, which may provide a good modelling system on structural evolution of metal NPs with different stages, and explore the relationship of processing-performance-structure [7].

\section{References:}

[1] L.D. Shao et al. Angew. Chem. Int. Ed. 52 (2013), DOI: 10.1002/anie.201207362.

[2] L.D. Li et al. ChemCatChem 4 (2012), p. 1764.

[3] C.M. Li et al. J. Mater. Chem. A 1 (2013), p. 2461.

[4] B.S. Zhang et al. Chem. Commun. 47 (2011), p. 10716.

[5] L.D. Shao et al. Angew. Chem. Int. Ed. 50 (2011), p. 10231.

[6] B.S. Zhang et al. Chem. Eur. J. 17 (2011), p. 12877.

[7] This work is supported by the IMR SYNL-T.S. Kê Research Fellowship, the National Natural Science Foundation of China (No. 21203215), and the China Postdoctoral Science Foundation (2012M520652).
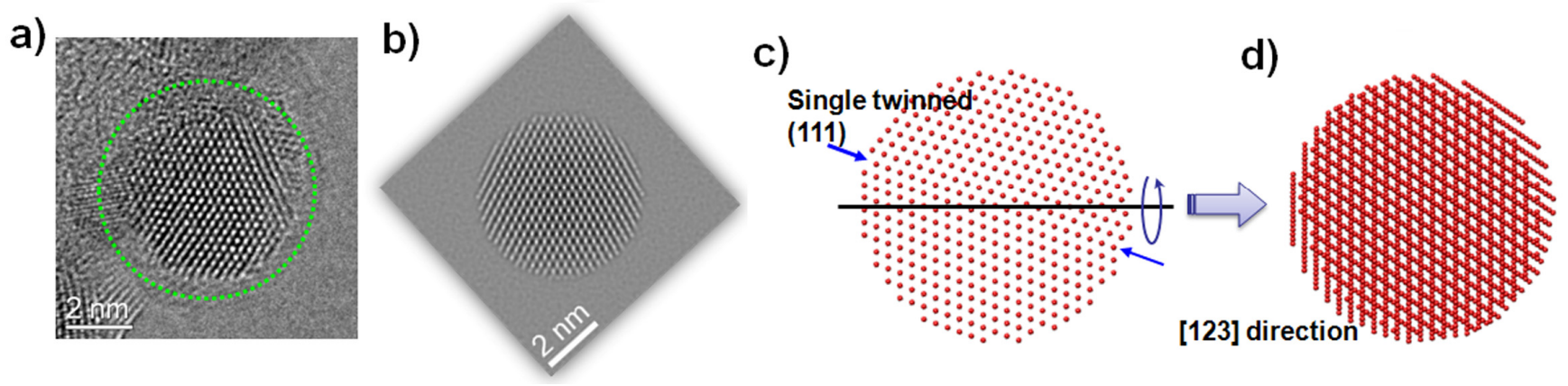

Figure 1. HRTEM (a) and simulated (b) images of Pd NP supported on carbon black, and the simulated model (c, d) of single twined Pd NP. If around an axis (black line) in (c), the [123] projection (d) can be obtained.
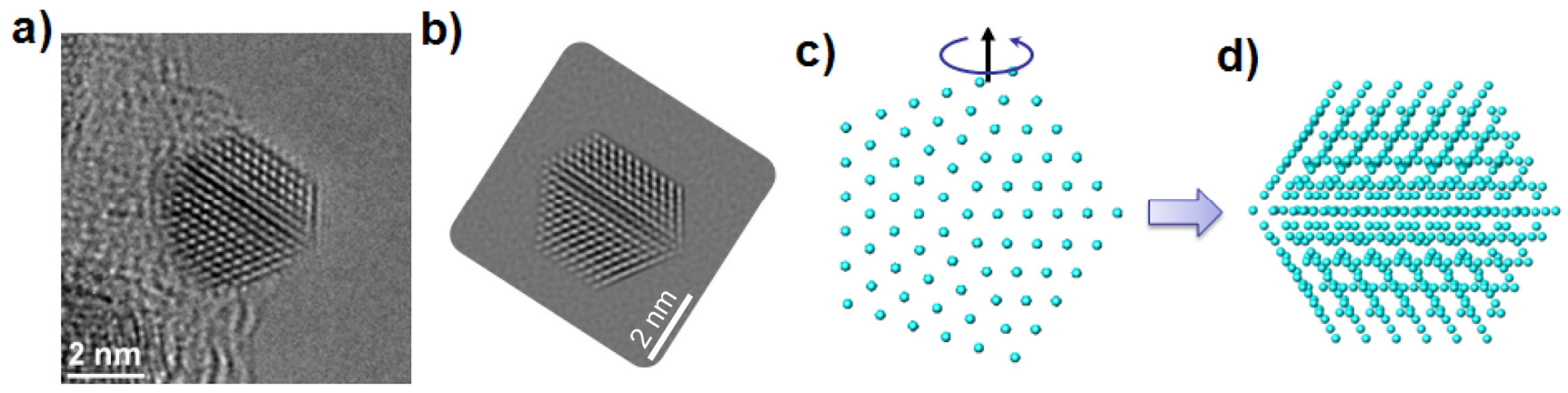

Figure 2. HRTEM (a) and simulated (b) images of $\mathrm{Au}$ NP supported on active carbon, and the simulated model (c, d) of multiply-twinned (truncated decahedron) Au NP. If around an axis (black arrow) in (c), the corresponding projection (d) with (a) can be obtained. 